\title{
INFLUENCE OF TERRAMYCIN ON THE COURSE OF EXPERIMENTAL OCULAR TUBERCULOSIS* COMPARATIVE ACTIVITY OF TERRAMYCIN, STREPTOMYCIN, AND BOTH ANTIBIOTICS COMBINED
}

BY

\author{
GIUSEPPE LEPRI AND STEFANO CAPALBI
}

From the Eye Clinic of the University of Pisa, Italy

(Director, Professor Filippo Caramazza)

AlThOUGH the broad antimicrobial spectrum of terramycin, the antibiotic recently isolated (Finlay and others, 1950), has now been sufficiently delimited, its influence in tubercular infections has not been established with any certainty.

Preliminary research has shown that the Mycobacterium tuberculosis has, in vitro, a certain degree of resistance to the action of the antibiotic. This notwithstanding, the earliest experimental research on tuberculous infection in animals (guinea-pigs and mice) has shown that the drug has a therapeutic effect (Steenken and Wolinsky, 1949), although it was possible to use only very small doses in the guinea-pig because of the toxic reaction produced by terramycin in this animal.

The report of Hobby and others (1950) on the therapeutic effect exerted by terramycin on experimental infections in mice seems to indicate that a dose of $7.1 \mathrm{mg}$. terramycin per day, administered subcutaneously to mice, is sufficient to produce an inhibiting effect on experimental tuberculosis.

As it seemed desirable to continue researches into the influence of this antibiotic in tubercular infection, we accepted the invitation of the Pfizer Company to investigate its action in experimental ocular tuberculosis.

We had to choose whether it would be better to induce the infection in normal animals or in animals allergized by a previous bacterial infection in another part of the organism. Although this second method causes the ocular tubercular infection to follow a more gradual course, and does, in fact, approximate more closely to the course of the clinical ocular infection, it does not lend itself so well to an evaluation of the effectiveness of an anti-tubercular drug because of the basic influence exerted by the reaction of the organism. Therefore we decided to conduct the experiment on normal, non-allergized animals (rabbits).

\section{Technique}

Into thirty male rabbits having an average weight of $1.8 \mathrm{~kg}$. we introduced, into the anterior chamber, $0.1 \mathrm{ml}$. of a suspension of Koch's bacilli (human variety), obtained by diluting in normal saline physiological solution a tuberculous patina

* Received for publication August 14, 1951. 
from Petragnani's culture medium, in the proportion of $1 \mathrm{mg} . / \mathrm{ml}$. Into the eyes of the animals thus infected we then instilled for three consecutive days a drop of atropine; all signs of ocular traumatic irritation generally disappeared by the second day.

Starting on the fourth day (that is, exactly $72 \mathrm{hrs}$ after the infection), the animals were divided into four groups:

I. Controls: no treatment-six animals.

II. Treated with streptomycin-eight animals.

III. Treated with terramycin - eight animals.

IV. Treated with streptomycin and terramycin combined-eight animals.

Route of Administration and Dosage.-Streptomycin was administered by the parenteral route in daily doses of $100 \mathrm{mg}$. (two $50-\mathrm{mg}$. intramuscular injections at 8 a.m. and 8 p.m.)*.

Terramycin was administered intramuscularly for ten consecutive days, in doses of $100 \mathrm{mg}$. per day per animal. The terramycin powder (hydrochloride) was dissolved in water and the solution adjusted to a $\mathrm{pH}$ of 6.5 to 7 by the addition of sodium chloride and sodium borate. The drug was given in three daily doses, administered at 9 a.m. (25 mg.), 4 p.m. (25 mg.), and 11 p.m. (50 mg.).

We noted that the parenteral introduction of terramycin causes in the animals a certain degree of motor excitation, which disappears rapidly, and at the point of injection we saw a certain degree of necrosis. This fact, together with the loss of weight and lack of appetite observed in the animals after the first few days of treatment, induced us, after 10 days of uninterrupted parenteral therapy, to continue the administration of terramycin by the oral route, in the same $100-\mathrm{mg}$ doses per day per animal. In order to promote as constant as possible an assimilare tion of the drug, the solution of terramycin was mixed with some solid food? (bread). This promptly caused the animals to resume their normal weight. Such oral treatment was continued without interruption for 20 days, thus bringing the total period of treatment with the antibiotic to 30 days. On that date all treatment was discontinued.

\section{Course of Infection}

I. Control Animals.-Taking an average of the times at which the various tubercular manifestations appeared in the individual animals of this group, we observed the first signs of specific phlogosis (conjunctival hyperaemia and iritic hyperaemia) about the 12th to 13th day of the infection. The appearance of the nodules on the iris, as we could observe under the biomicroscope, occurred about the 16th to 17th day, and was followed by the establishment of marked congestion and oedema of the iris during the period from the 18th to the 24th day. Between the 20th and 22nd days, the cornea began to be invaded by pannus, which, starting from the upper sector, subsequently extended to almost the entire membrane. The eyeball appeared in incipient buphthalmos during the fourth week, and, during the fifth week, a process of panophthalmitis set in, leading in four animals out of six to the perforation of the bulbus between the 45th and

\footnotetext{
* We preferred to administer the streptomycin in two daily doses rather than in a single dose; to obtain a concentration in the blood as constant as possible.

+ Although a sufficiently constant level of terramycin in the blood can also be obtained by means of a single daily administration, we here also favoured a divided dosage, to ensure the most constant levels possible.
} 
52nd days of the infection. This was followed by the regressive phenomena, finally resulting in atrophy of the eyeball. In the two animals, in which such perforation did not occur, the outcome was likewise total atrophy of the bulbus.

II. Animals treated with Streptomycin.-Confirming previous experience, the infection assumed a rather favourable course. Although the first signs of inflammation of the iritic membrane appeared somewhat later than in the control animals (on the 14th to 15th day), the intensity of the iridic congestion and oedema and the number and diffusion of the tuberculous nodules were found to be markedly reduced. The corneal pannus did not appear in all the animals, and, where it did appear, was limited to the periphery of the membrane. In none of the animals did the phlogistic process reach the stage of tuberculous panophthalmitis, and the outcome consisted of atrophy of the iris, more or less marked posterior synechia, and, now and then, partial pupillary occlusion. The bulbus never appeared in a state of buphthalmos. Once the infection had ceased, the animals visibly retained a certain degree of visual acuity. All signs of phlogosis disappeared on the 50th day after the infection.

III. Animals treated with Terramycin.-The initial course was decidedly more favourable than in the untreated control animals; the first signs of inflammation generally appeared after the 15th day of infection, and these were attenuated and limited. There was also a reduction in the number and size of the specific nodules appearing on the iris. The subsequent course reflected a certain attenuation, and, above all, a delayed development, which was clearly visible up to the 25 th to 28 th day of the infection. On the other hand, after this date, we noted a definite aggravation of the specific symptoms which, when the treatment was suspended (on the 30th day), had become nearly as intense as those in the control animals. The final outcome was perforation and atrophy of the bulbus in half the cases, and atrophy without passing through the stage of perforation in the remainder. It should be noted that a similar result was seen later in the control animals, so that the phlogosis, although more attenuated on the whole, was of longer duration.

IV. Animals treated with Terramycin and Streptomycin combined.-On the whole, the course of the infection was similar to that observed in the animals treated with streptomycin only.

It is, however, worthy of note that the first signs of phlogosis appeared much later (after the 20th day in all the animals). The subsequent course and the ultimate inflammation were similar to those in the second group. The tuberculous nodules were scarce, the congestion and oedema of the iris limited, and the pannus on the cornea confined to the upper sector of the membrane. The final outcome was corneal nubeculae, partial atrophy of the iris, posterior synechia, and, at times, an occluded pupil. All signs of inflammation disappeared on the 50th to 55th day in all the animals.

\section{Discussion}

A comparison of the action of terramycin in experimental ocular tuberculosis with that exerted by streptomycin under the same conditions and in identical doses shows that the latter has a more favourable influence upon the course of the infection. However, it seems to us that terramycin also 


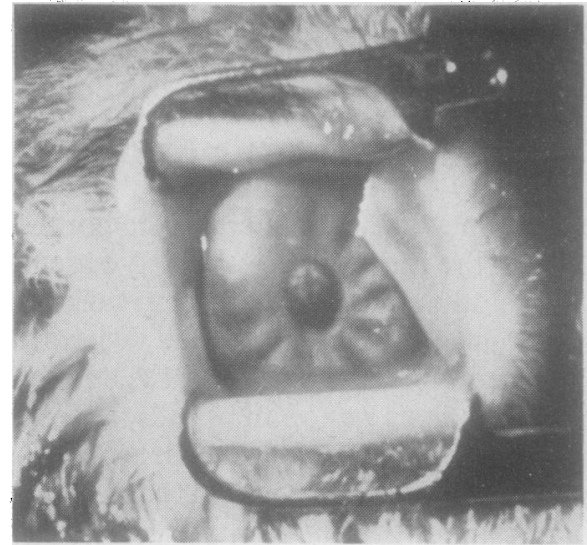

Fig. 1.-Untreated control eye (20th day from infection). Beginning of corneal pannus; congestion and oedema of iris; tuberculous nodules on surface of iris (Rabbit No. 5).

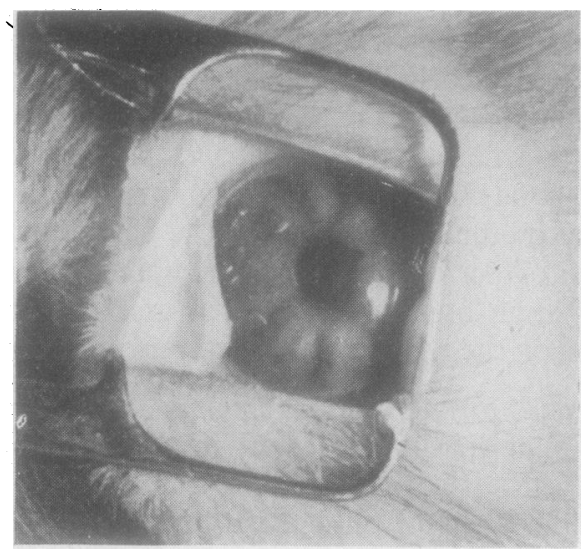

FIG. 3.-Terramycin-treated eye (20th day from infection, 17th day from start of treatment). Similar appearance to Fig. 2 (Rabbit No. 16).

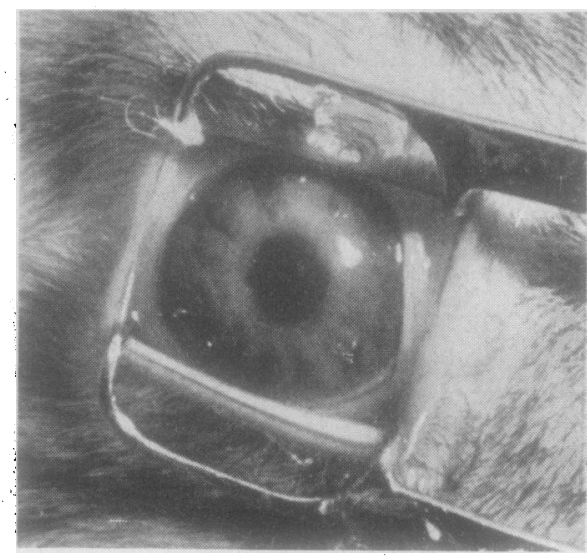

Fig. 2.- Streptomycin-treated eye (20th day from infection, 17th day from start of treatment). No pannus; slight congestion and oedema of iris; small tuberculous nodules on surface of iris (Rabbit No. 11).

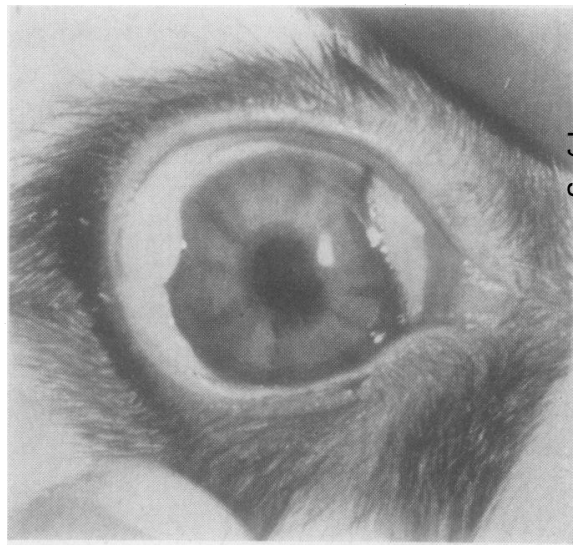

FIG. 4.-Streptomycin/terramycin-treated eye (20th day from infection, 17th day from start of treatment). Only slight congestion of iris nodules not yet visible on surface of iris (Rabbit No. 26).

has a certain measure of effectiveness against tuberculosis, as evidenced by the delayed appearance of tuberculous manifestations in the iris and of the other classic symptoms of inflammation. On the other hand, we were able to observe that the delaying effect on tuberculosis is considerably reduced after the 20th or 25th day of treatment, as if the bacteria had acquired a certain degree of immunity to the antibiotic, and that it ceases completely after the suspension of the treatment, whereas the effect continues without any flare-up in animals treated with streptomycin. 


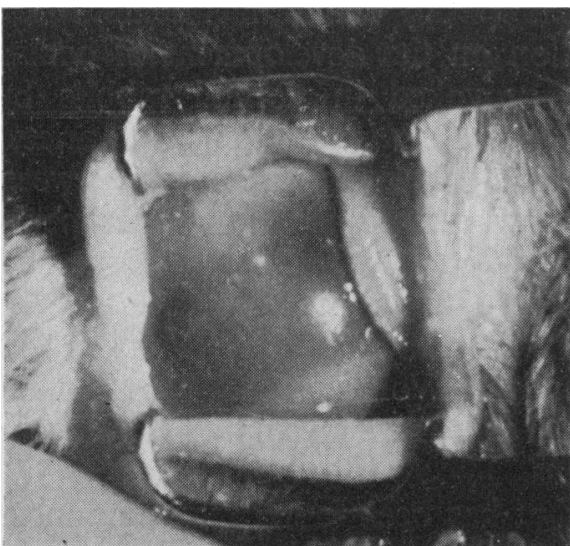

FIG. 5.-Untreated control eye (28th day from infection). Cloudiness of cornea and evident corneal pannus; buphthalmos; appearance of iris not visible; incipient panophthalmitis (Rabbit No. 3).

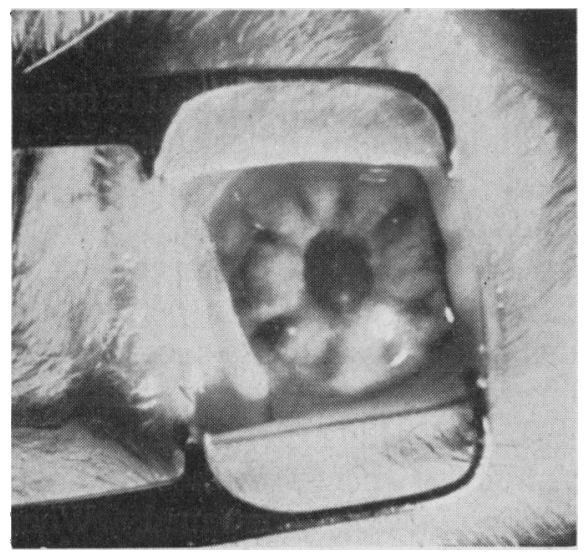

Fig. 7.-Terramycin-treated eye (28th day from infection, 25th day from start of treatment). Beginning of corneal pannus; marked congestion and oedema of iris; large tuberculous nodules clearly visible (Rabbit No. 19).

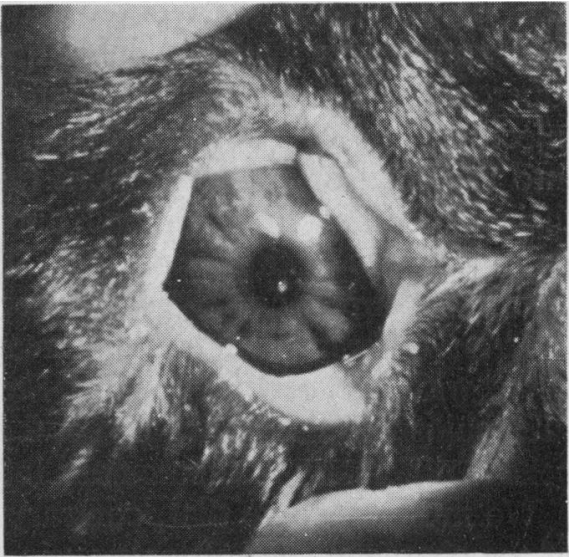

FIG. 6.-Streptomycin-treated eye (28th day from infection, 25th day from start of treatment). Transparent cornea; congestion and oedema of iris; tuberculous nodules visible on surface of iris (Rabbit No. 13).

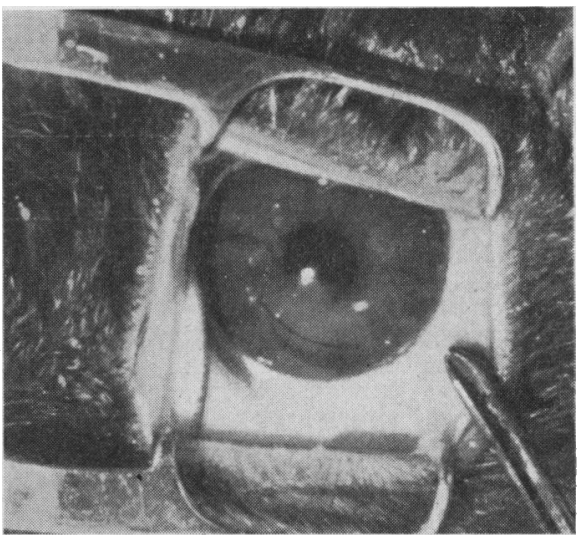

FIG. 8.-Streptomycin/terramycin-treated eye (28th day from infection, 25th day from start of treatment). Transparent cornea; slight congestion and oedema of iris; small tuberculous nodules visible on surface of iris (Rabbit No. 27).

The combined terramycin-streptomycin treatment resulted in the first signs of tubercular phlogosis appearing after a still longer interval, but the final evolution was no better than in animals treated with streptomycin only,

Thus we can definitely affirm that terramycin has some measure of antitubercular activity, which could be detected even in the severe experimental infection provoked in our experiments.

We feel that it would be useful to compare the activity of terramycin with 
that of aureomycin. Woods and others (1950) could not demonstrate that aureomycin had any inhibiting action on the development of ocular tuberculosis in experimental infections provoked in normal rabbits by injections of a suspension of myco. tuberculosis (human variety) into the anterior chamber. But there was a substantial difference between their experimental conditions and our own, since in their experiments treatment was instituted on the 12th day, when the first modifications had already occurred, and the doses were smaller $(20 \mathrm{mg}$. per $\mathrm{kg}$.).

These different results from treatment with aureomycin and terramycin agree with the observations of Steenken and Wolinsky (1949) in general tuberculosis of the guinea-pig, and confirm that aureomycin and terramycin behave differently in tuberculous infections.

We must conclude that, contrary to what has been found regarding most of the characteristics of terramycin and aureomycin (antibacterial spectrum, and effects on micro-organisms, both Gram-positive and Gram-negative, as well as absorption, excretion, etc.), which are almost identical, a different action exists with respect to tuberculous infëctions. Here terramycin-at least under the conditions of our investigation-displays a definite degree of activity, though this is slight compared with that of streptomycin.

\section{Summary}

The influence of terramycin on the course of experimental ocular tuberculosis induced by injections of Koch's bacilli into the anterior chamber has been investigated, and comparison made with the activity of streptomycin̄ under the same experimental conditions and in the same dosages.

The action of terramycin was found to be decidedly inferior to that of streptomycin, but there was definite evidence that terramycin delayed the appearance of the first signs of tuberculous phlogosis and the succession of the other classic symptoms of inflammation. After the suspension of treatment there was a prompt recurrence of phlogosis, and the final outcome was the same as in the control animals, whereas in those animals where streptomycin was used, the inhibiting activity continued. When both antibiotics were used in combination, a very slight advantage was observed over the effect of streptomycin used alone.

A comparison of the results obtained by the authors with those obtained in a previous investigation by Woods and others (1950), in which aureomycin was used, although under different experimental conditions, reveals that, whereas aureomycin is incapable of inhibiting the course of experimental tuberculous infections, terramycin - at least under the experimental conditions observed by the authors-has a slight but definite inhibitory activity.

\section{REFERENCES}

FinLAY, A. C., and ten others (1950). Science, 111, 85.

Hobby, G. L., DougherTy, N., Lenert, T. F., Hudders, E., and Kiseluk, M. (1950). Proc. Soc. exp. Biol., N.Y., 73, 503.

Steenken, W., JNR., and WolinsKy, E. (1949). Amer. Rev. Tuberc., 59, 221.

(1950). Ann. N.Y. Acad. Sci., 53, 309.

Woods, A. C., Wood, R. M., and NAQUIN, H. A. (1950). Arch. Ophthal., Chicago, 43, 845. 